\title{
Expression of CD31 in Mycosis Fungoides
}

\author{
ALINA JANKOWSKA-KONSUR ${ }^{1}$, CHRISTOPHER KOBIERZYCKI ${ }^{2}$, \\ JEDRZEJ GRZEGRZOLKA ${ }^{2}$, ALEKSANDRA PIOTROWSKA ${ }^{2}$, AGNIESZKA GOMULKIEWICZ ${ }^{2}$, \\ NATALIA GLATZEL-PLUCINSKA ${ }^{2}$, MATEUSZ OLBROMSKI ${ }^{2}$, \\ MARZENNA PODHORSKA-OKOLOW ${ }^{2}$, JACEK C. SZEPIETOWSKI ${ }^{1}$ and PIOTR DZIEGIEL ${ }^{2,3}$
}

\author{
Departments of ${ }^{1}$ Dermatology, Venereology and Allergology and \\ ${ }^{2}$ Histology and Embryology, Wroclaw Medical University, Wroclaw, Poland; \\ ${ }^{3}$ Department of Physiotherapy, University School of Physical Education, Wroclaw, Poland
}

\begin{abstract}
Background/Aim: Mycosis fungoides (MF) is the most common primary cutaneous T-cell lymphoma (CTCL) characterized by malignant proliferation of mature $T$ lymphocytes and primary skin involvement. Recent reports suggest that angiogenesis may play a role in the growth and spread of this malignancy. Cluster of differentiation 31 (CD31) is a protein classified into the Ig-superfamily of cell adhesion molecules, expressed on endothelial cells and majority of hematopoietic non-erythroid cells. The aim of our study was to explore the role of angiogenesis in MF. Materials and Methods: We evaluated the expression of CD31 in relation to clinicopathological data and potential impact on patients' outcome in MF utilizing immunohistochemistry (IHC) and western blot (WB) techniques in 73 and $19 M F$ and 21 and 4 control samples, respectively. Results: In the IHC study, statistical analysis revealed significantly higher CD31 expression in MF compared to the controls $(p<0.0001)$ with highest expression in advanced stages vs. early ones and controls $(p<0.0001$ for both). In regard to skin involvement, expression was also elevated in more infiltrative (T3, tumors) and in more extensive (T4, erythroderma) cutaneous lesions compared to less infiltrative and limited skin lesions (T1, T2, patches and/or plaques) ( $p<0.01$ for both). Regarding the extracutaneous spread, higher CD31 expression correlated with nodal involvement (N1-3 vs. NO; $<<0.0001)$. In the WB study, statistical analysis revealed significantly higher CD31 expression only in advanced vs. early stage of $M F(p<0.05)$. In regard to skin involvement, expression was also elevated in $T 3$ and $T 4$ as compared $T 1+T 2(p=0.08, p<0.05$; respectively).
\end{abstract}

Correspondence to: Christopher Kobierzycki, MD, Ph.D., Department of Histology and Embryology, Wroclaw Medical University, Chalubinskiego 6a, 50-368 Wroclaw, Poland. Tel: +48 717841682, Fax: +48 717840082, e-mail: ch.kobierzycki@gmail.com

Key Words: Mycosis fungoides, primary cutaneous T-cell lymphoma, angiogenesis, CD31.
Higher CD31 expression correlated with nodal involvement (N1-3 vs. N0; $p<0.01)$. A strong significant correlation between CD31 expression at the protein level analyzed by IHC and WB was noticed $(r=0.802, p<0.0001)$. Moreover, strong positive correlations between IHC expression of CD31 and podoplanin (PDPN; $r=0.582, p<0.0001)$, vascular endothelial growth factor $C$ (VEGFC; $r=0.332, p<0.01)$ and Ki-67 $(r=0.330$, $p<0.01)$ were disclosed. Conclusion: Expression of CD31 in MF skin biopsies provides new evidence for the role of angiogenesis in the progression of MF. Additionally, the new data revealed prompts for further research on potential use of CD31 as a new marker of the disease advancement, as well as the target of new therapeutic strategies.

Mycosis fungoides (MF) is the most common primary cutaneous T-cell lymphoma (CTCL) characterized by malignant proliferation of mature $\mathrm{T}$ lymphocytes with the Th2 phenotype. The disease primarily develops in the skin, classically evolving from patches through infiltrated plaques to tumors. In approximately $30 \%$ of cases, extracutaneous dissemination to the lymph nodes and distant organs is observed. Tumor, node and metastasis blood (TNMB) classification illustrates classical progression of the disease (1, 2). Recent years have brought significant progress in research on MF; however, the mechanisms of the disease development and progression are still not fully elucidated. Several lines of evidence suggest that, similarly to solid tumors, angiogenesis may play an important role in the growth and spread of MF (3-9). Angiogenesis is a multistep process of new capillaries formation from existing vascular network through the extension and branching in response to stimulation by proangiogenic factors. It is an important component of physiological processes (i.e., during pregnancy, menstrual cycle and tissue regeneration) and it, also, plays a fundamental role in tumor growth and dissemination (10-13).

Cluster of differentiation 31 (CD31), also known as plateletendothelial cell adhesion molecule-1 (PECAM-1), is a transmembrane glycoprotein type I, a member of the Ig- 
superfamily of cell adhesion molecules (14). CD31 has a molecular weight of $130 \mathrm{kDa}$ and is composed by 6 extracellular Ig folds involving $\mathrm{N}$ - and O-linked glycosylation sites (15-16). CD31 expression is observed on endothelial cells and a majority of hematopoietic non-erythroid cells, including platelets, neutrophils, T and B cell subsets (14). Accumulated data indicate the role of CD31 in vascular barrier function integration, mechanical stress sensing, apoptosis prevention and angiogenesis (11). It has been also widely used as a marker of angiogenesis in immunohisto-chemistry studies (17).

Several studies concerning angiogenesis in MF have been published over the last years; however, detailed analysis of the CD31 expression in MF, utilizing immunohistochemistry (IHC) and western blot (WB), has not been published yet. Therefore, the aim of our study was to evaluate the expression of CD31 in MF in relation to clinicopathological data and potential impact on patients' outcomes in MF.

\section{Materials and Methods}

Specimens. The IHC study was performed on 73 paraffin blocks obtained from the patients with MF diagnosed and treated between years 1994-2015 in the Department of Dermatology, Venereology and Allergology of Wroclaw Medical University, Poland. The diagnosis was established and re-evaluated based on clinical, histopathological and IHC examinations, according to the World Health Organization (WHO) classification (2008) (1). The staging was assessed according to TNMB system (International Society for Cutaneous Lymphomas/ European Organization for Research and Treatment of Cancer; ISCL/EORTC) revision) also presented in our previous study $(2,18,19)$. In order to analyze potential differences between benign and malignant skin conditions, the control group comprised of 19 paraffin blocks obtained from aged- and sex-matched patients with benign inflammatory dermatoses (16 with lichen planus, 3 with eczema disseminatum). Additionally, samples from 21 patients with MF and 4 with benign dermatoses skin biopsies were also collected and stored in $-80^{\circ} \mathrm{C}$ in order to perform WB analyses. The experiment was performed in accordance to ethical standards and approved by the Ethics Committee of Wroclaw Medical University (decision no. KB 574/2011). Patients' dermographic and clinicopathological characteristics are presented in Table I.

Immunohistochemistry (IHC). All reactions were performed on 4- $\mu \mathrm{m}-$ thick paraffin sections. In order to examine the CD31 expression, a protocol using Dako Autostainer Link48 (Dako, Glostrup, Denmark) was introduced. Deparaffinization, rehydratation and antigen retrieval was conducted by boiling in EnVision FLEX Target Retrieval Solution ( $\mathrm{pH}=9,20 \mathrm{~min}, 7^{\circ} \mathrm{C}$; Dako) using Pre-Treatment Link platform (Dako). Activity of endogenous peroxidase was blocked by a 5-min incubation in EnVision FLEX Peroxidase-Blocking Reagent (Dako). Sections were incubated with the primary monoclonal mouse anti-human CD31 antibody (clone JC70A, RTU; Dako) for $20 \mathrm{~min}$ at room temperature (RT). Following this EnVision FLEX /HRP treatment, secondary antibodies were applied (20 min at RT; Dako). Diaminobenzidine ((DAB); Dako) was utilized as the peroxidase substrate and the sections were incubated for $10 \mathrm{~min}$ at RT. All slides were counterstained with EnVision FLEX Hematoxylin (Dako). Subsequently, the preparations were mounted in Mounting Medium (Dako).
Table I. Patients' clinicopathological data.

\begin{tabular}{|c|c|c|c|c|}
\hline \multirow{3}{*}{$\begin{array}{l}\text { Mean age (range) } \\
\text { Parameters }\end{array}$} & \multicolumn{2}{|c|}{ IHC $(n=73)$} & \multicolumn{2}{|c|}{ WB $(n=21)$} \\
\hline & 58.82 & $5(19-81)$ & 56.4 & $1(31-77)$ \\
\hline & $\mathrm{N}$ & $\%$ & $\mathrm{~N}$ & $\%$ \\
\hline \multicolumn{5}{|l|}{ Gender } \\
\hline Male & 46 & 63.89 & 12 & 57.14 \\
\hline Female & 27 & 37.50 & 9 & 42.86 \\
\hline \multicolumn{5}{|l|}{ Tumor size } \\
\hline $\mathrm{T} 1$ & 20 & 27.40 & 6 & 28.57 \\
\hline $\mathrm{T} 2$ & 22 & 30.14 & 3 & 14.29 \\
\hline $\mathrm{T} 3$ & 15 & 20.54 & 6 & 28.57 \\
\hline $\mathrm{T} 4$ & 16 & 21.92 & 6 & 28.57 \\
\hline \multicolumn{5}{|l|}{ Lymph nodes } \\
\hline NO & 42 & 58.33 & 9 & 42.86 \\
\hline $\mathrm{N} 1, \mathrm{~N} 2, \mathrm{~N} 3$ & 31 & 43.06 & 12 & 57.14 \\
\hline \multicolumn{5}{|l|}{ Metastasis } \\
\hline M0 & 72 & 98.63 & 21 & 100.00 \\
\hline M1 & 1 & 1.37 & 0 & 0 \\
\hline \multicolumn{5}{|l|}{ Blood involvement } \\
\hline B0 & 69 & 94.52 & 20 & 95.24 \\
\hline $\mathrm{B} 1$ & 4 & 5.48 & 1 & 4.76 \\
\hline \multicolumn{5}{|l|}{ pTNM } \\
\hline I A & 19 & 26.03 & 6 & 28.57 \\
\hline I B & 16 & 21.92 & 2 & 9.52 \\
\hline II A & 6 & 8.22 & 1 & 4.76 \\
\hline II B & 11 & 15.07 & 5 & 23.81 \\
\hline III & 15 & 20.55 & 6 & 28.57 \\
\hline IV A & 5 & 6.85 & 1 & 4.76 \\
\hline IV B & 1 & 1.36 & 0 & 0 \\
\hline \multicolumn{5}{|c|}{$\begin{array}{l}\text { Clinical advancement } \\
\text { Early }\end{array}$} \\
\hline (stage IA-IIA) & 46 & 63.01 & 12 & 57.14 \\
\hline Advanced & & & & \\
\hline (stage IIB-IVB) & 27 & 36.99 & 9 & 42.86 \\
\hline
\end{tabular}

IHC, Immunohistochemistry; WB, western blot.

Evaluation of IHC reactions. All sections were evaluated using a BX-41 light microscope (Olympus, Tokyo, Japan) by two pathologists who were blinded to the patients' clinical data. In doubtful cases, a re-evaluation with a double-headed microscope was performed until a consensus was achieved. CD31 expression was observed in the cytoplasm of endothelial cells. Initially, the sections were first scanned at low power $(\times 40$ and $\times 100$ magnification) to identify the hot-spots (areas of potentially highest microvessel density (MVD) count). Subsequently, these areas were examined under $\times 200$ magnification using a Chalkley Point Array graticule (Pyser Sgi., Edenbridge, UK). The Chalkley count was regarded as the number of grid points that hit stained vessels. An average score was determined for three hot-spots.

Western blot (WB). Frozen samples were thawed in CellLytic MT Cell Lysis Solution (SigmaAldrich, Munich, Germany) with the addition of protease inhibitors, Benzonase at 50U/ $\mu$ l (Merck; Millipore, Bedford, MA, USA) and 0.2 mM phenylmethanesulfonyl fluoride ((PMSF); SigmaAldrich). Protein concentrations of whole cell lysates were determined by bicinchoninic acid (BCA) assay 

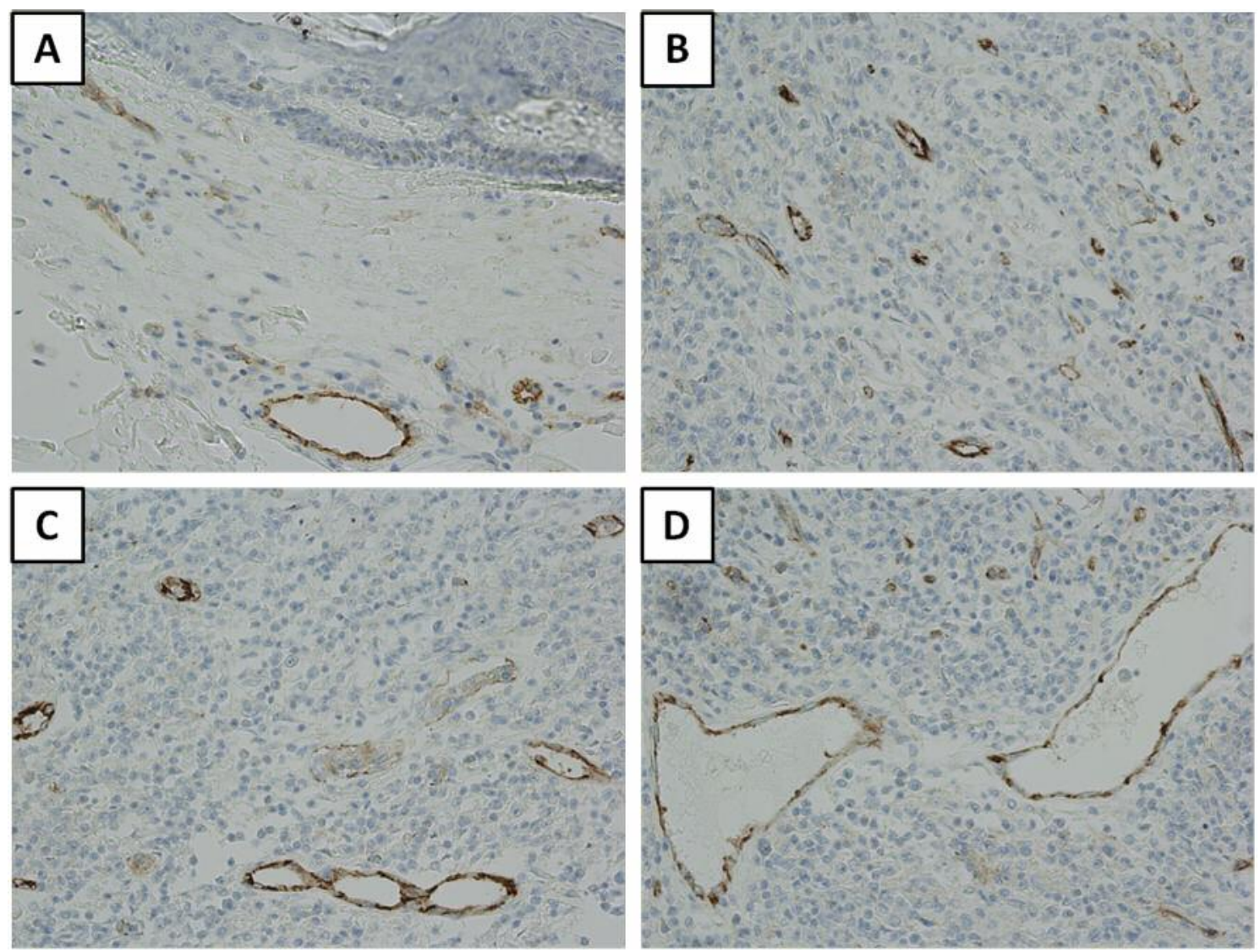

Figure 1. Immunohistochemical expression of CD31 in cytoplasm of endothelial cells in benign dermatosis (A, control) and in mycosis fungoides patients $(B-D)$. Magnification $\times 200$.

(Pierce, Rockford, IL, USA). Equal amounts of total protein $(30 \mu \mathrm{g})$ were mixed with sample buffer and dithiothreitol (DTT) and resolved by sodium dodecyl sulfate-polyacrylamide gel electrophoresis (SDS-PAGE). After the completion of the electrophoresis, samples were transferred to polyvinylidene difluoride (PVDF) membranes (Immobilon; Millipore) and incubated in a $4 \%$ bovine serum albumin (BSA) solution in tris-buffered saline (TBS) with the addition of $0.1 \%$ Tween-20. Subsequently, membranes were incubated with mouse anti-human CD31 antibody at 1:200 (66065-1-Ig; Proteintech Europe, Manchester, UK) overnight at $4{ }^{\circ} \mathrm{C}$. At the end, the membranes were treated with the secondary antibody specific for the primary antibody (Jacksons Immunoresearch, Suffolk, UK) for $1 \mathrm{~h}$, rinsed and incubated with the Immun-Star-HRP Chemiluminescent Substrate (Bio-Rad, Hercules, CA, USA). Protein quantifications were based on the total protein normalization with the use of the TGX Stain Free FastCast Acrylamide Kit (Bio-Rad). Protein separation was visualized on the gels after electrophoresis and analyzed by using Image Lab 5.0 Software and ChemiDock MP System (Bio-Rad).

Statistical analysis. Shapiro-Wilk test was used to evaluate the normality assumption of examined groups. To compare the differences between the expression of examined markers in all patients' pairs of groups and clinicopathological data, the unpaired t-test and Mann-
Whitney test were used. To compare the differences between more than two groups, the Kruskal-Wallis and Dunn's multiple comparison test was performed. Additionally, the Spearman correlation test was used to analyze the existing correlations. The Kaplan-Meier method was used to construct survival curves. To evaluate the analysis of survival Mantel-Cox test was performed. A Cox proportional hazards model with forward stepwise selection was used to calculate univariate and multivariate hazard ratio (HR) for the study variables. All statistical analyses were performed using Prism 5.0 (GraphPad, La Jolla, CA, USA) and Statistica 10 (StatSoft Inc. Tulsa, OK, USA). The results were considered as statistically significant when $p<0.05$.

\section{Results}

Immunohistochemistry (IHC). Positive reactions for CD31 were disclosed in 71/73 of MF cases and in all of control samples. The CD31 expression was observed in cytoplasm of endothelial cells of blood vessels (Figure 1). Statistical analysis revealed that CD31 expression was significantly higher in MF as compared to controls ( $p<0.0001$; MannWhitney test; Figure 2A). In MF, the mean expression of the studied protein was significantly higher in advanced stages 

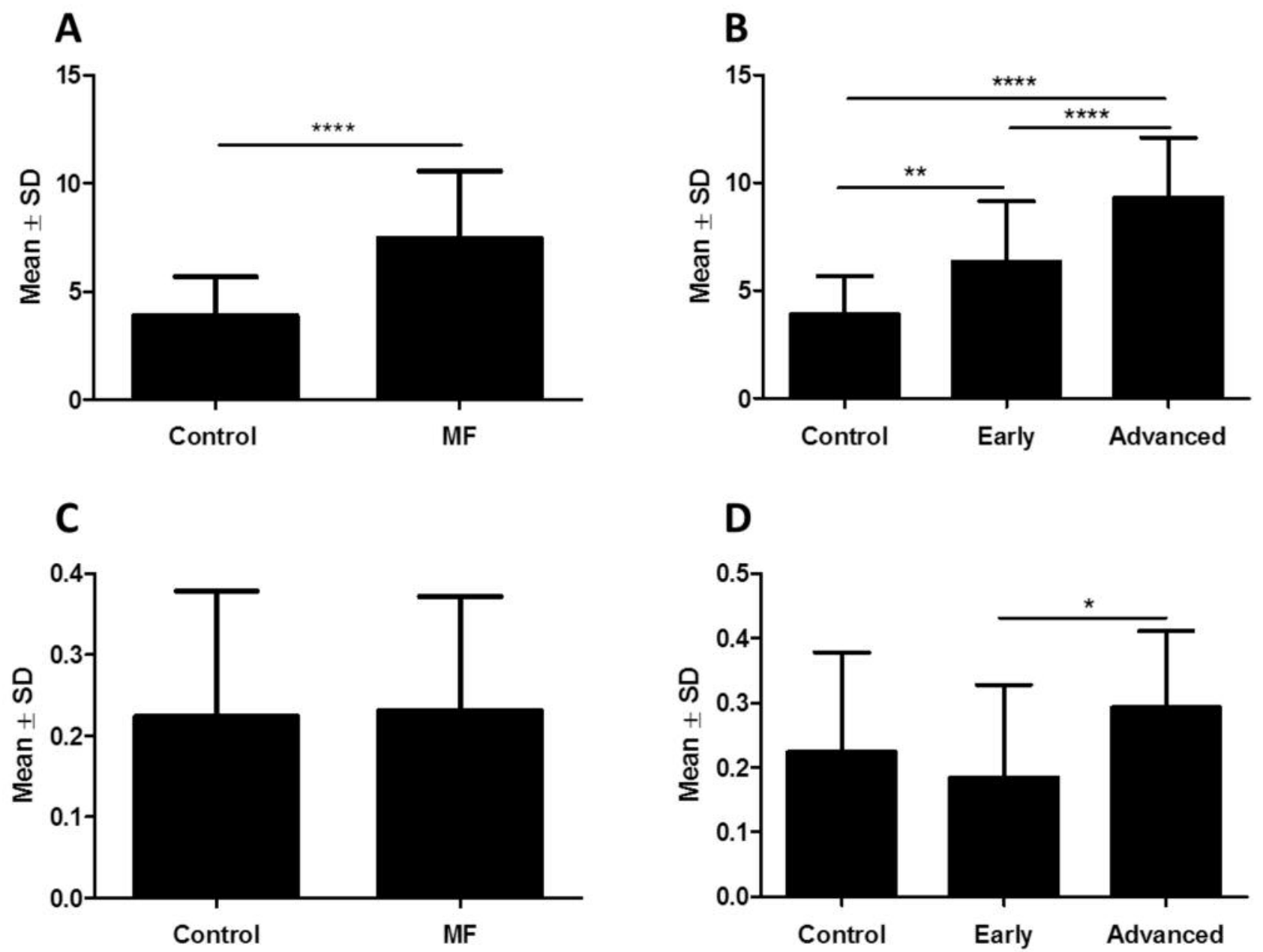

Figure 2. Comparison of $C D 31$ expression assessed with immunohistochemistry $(A, B)$ and western blot $(C, D)$ in regard to disease presence $(A, C)$ and clinical advancement $(B, D)$. MF, Mycosis fungoides; OD, optical density; SD, Standard deviation. *p<0.05, **p<0.01, ****p<0.0001, MannWhitney test.

than in early ones and controls $(p<0.0001$ for both) as well in early stages compared to controls $(p<0.01$; Mann-Whitney test; Figure 2B). CD31 expression was also elevated in more infiltrative cutaneous lesions ( $\mathrm{T} 3$, tumors) and in more extensive skin involvement (T4, erythroderma) as compared to less infiltrative and limited skin changes (T1, T2, patches and/or plaques) ( $p<0.01$ for both, Mann-Whitney test, Figure $3 \mathrm{~A}-\mathrm{B})$. Moreover, the infiltration of the skin changes had more important statistical significance than the extent of the lesions in early stages $(\mathrm{T} 1 \mathrm{~b}+\mathrm{T} 2 \mathrm{~b} v s$. T1 $\mathrm{a}+\mathrm{T} 2 \mathrm{a} ; p<0.01$, MannWhitney test, Figure 3C). Regarding the extracutaneous spread, higher CD31 expression was associated with nodal involvement (N1-3 vs. N0) $(p<0.0001$, Mann-Whitney test, Figure 3D). Additionally, strong significant positive correlation between expression of CD31 and previously described proteins, i.e. podoplanin (PDPN; $\mathrm{r}=0.582$, $p<0.0001$ ), vascular endothelial growth factor C (VEGFC; $\mathrm{r}=0.332, p<0.01)$ and Ki-67 $(\mathrm{r}=0.330, p<0.01)$, was noticed (Spearman's correlation test; Figure 4 and Table II) (19).

Western blot $(W B)$. To verify the results obtained by IHC reaction, we also investigated CD31 expression in frozen 21 $\mathrm{MF}$ and 4 control samples by WB (Figure 5). Analyzing MF group, we found significantly higher CD31 expression in advanced $v s$. early stages $(p<0.05$, Mann-Whitney test; Figure 2C-D). Regarding the skin involvement, we noted the same associations between the CD31 expression and the infiltration and extend of the skin lesions (T3 vs. T1+T2, T4 vs. T1+T2) as in IHC study ( $p=0.08, p<0.05$, respectively; Mann-Whitney test; Figure 6A-C). Similarly, analyzing metastases, we confirmed the correlation between elevated CD31 expression and nodal involvement (N0 vs. N1-3) $(p=0.0095$; Mann-Whitney test; Figure 6D). We noticed a strong significant correlation between CD31 expression at the 

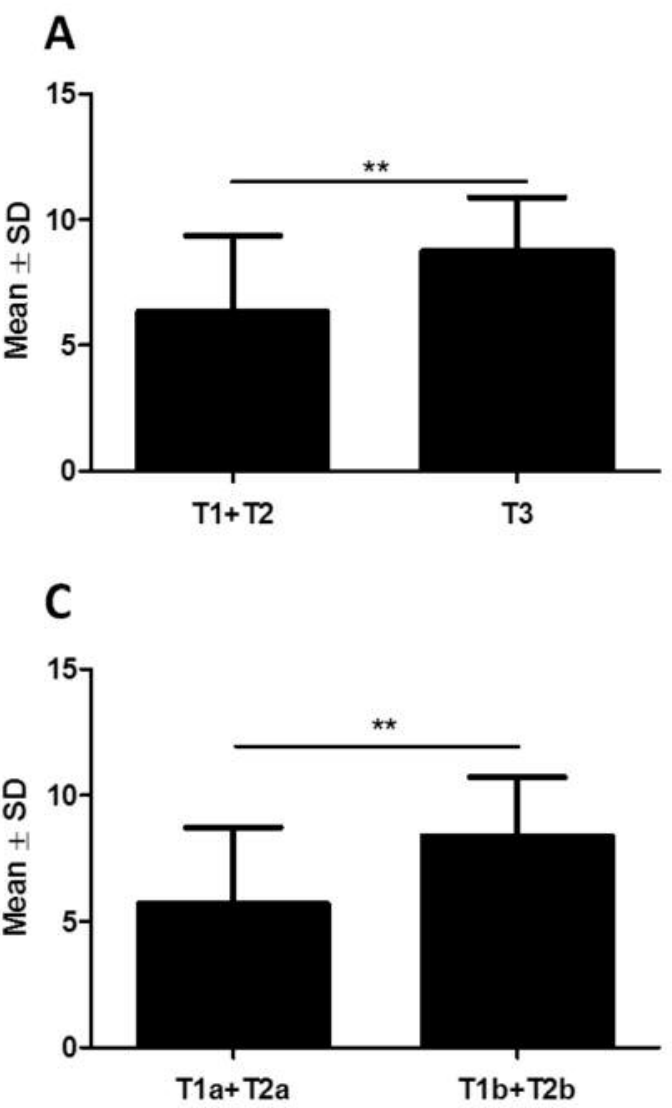

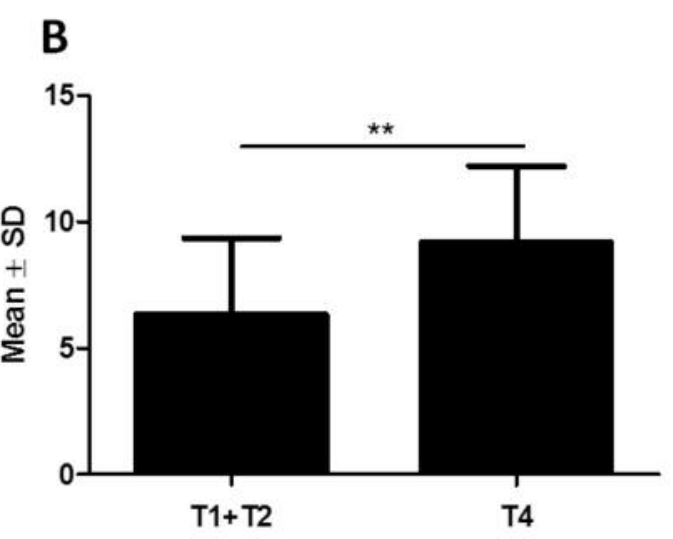

D

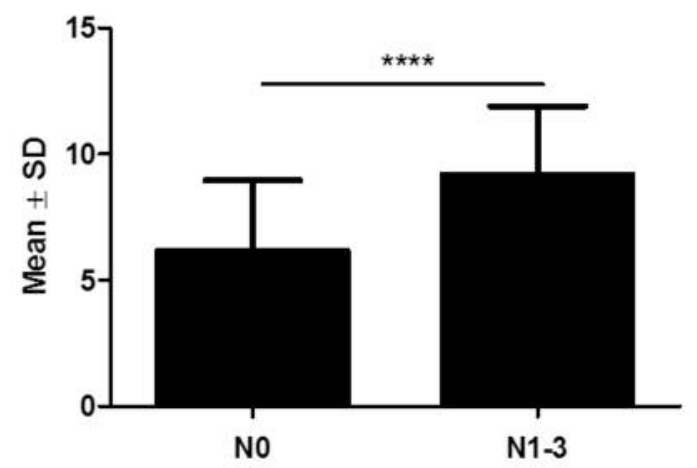

Figure 3. Comparison of CD31 expression assessed with immunohistochemistry (IHC) in regard to different stages of skin advancement (A-C) and nodal involvement $(D)$ in mycosis fungoides. SD, Standard deviation. ${ }^{* *} p<0.01, * * * p<0.0001$, Mann-Whitney test.

protein level assessed by IHC and WB $(r=0.8, p<0.0001$ Spearman's correlation test; Figure 4). Moreover, analysis of correlation of WB expression of previously studied proteins, i.e. PDPN, VEGFC and $\mathrm{Ki}-67$, with $\mathrm{CD} 31$ revealed no significant associations (Table II) (19).

Analysis of survival. A detailed summary was already been presented in previous reports on the expression of selected markers in CTCL, including MF subgroup (18-20). Regarding CD31 expression, univariate analysis using Mantel-Cox test did not reveal any significant associations in overall survival of MF patients, which may be explained by the relatively small patient cohort. (Table III). Additionally, no significant relationship between CD31 expression and age, gender and disease duration was noted (data not shown).

\section{Discussion}

Over the past decades, several reports have documented increased angiogenesis in CTCL, including MF (3-7, 21). Vacca et al. demonstrated increased angiogenesis measured by microvessel density (MVD) in skin biopsies obtained from patients with MF in regard to healthy skin controls (3). Furthermore, MVD was also significantly higher in cutaneous lesions $v s$. unchanged skin in MF cases and correlated with the advancement of skin disease. Similar results were published by Mazur et al., who evaluated MVD using antiCD34 antibodies in MF skin lesions (4). In the study of Jankowska-Konsur et al. MVD was significantly higher in advanced MF compared to early stages; however, there were no significant differences in MVD between early MF and either the healthy skin or parapsoriasis en plaque, a benign inflammatory dermatosis, transforming in MF, in some cases $(5,18)$. Interestingly, dermoscopy, non-invasive diagnostic method using magnifier $(\times 10-20)$ and a standardized light source, was also found to assess angiogenesis in skin lesions. In MF, Bosselia et al. have visualized a dotted pattern of blood vessels whose number correlated with the MVD as assessed by anti-CD34 antibodies (6). Recently, the expression of the key proangiogenic factor vascular endothelial growth factor A (VEGFA) has been observed in MF cells on both mRNA and protein level (7). Similarly, the expression of angiopoietin-2, 

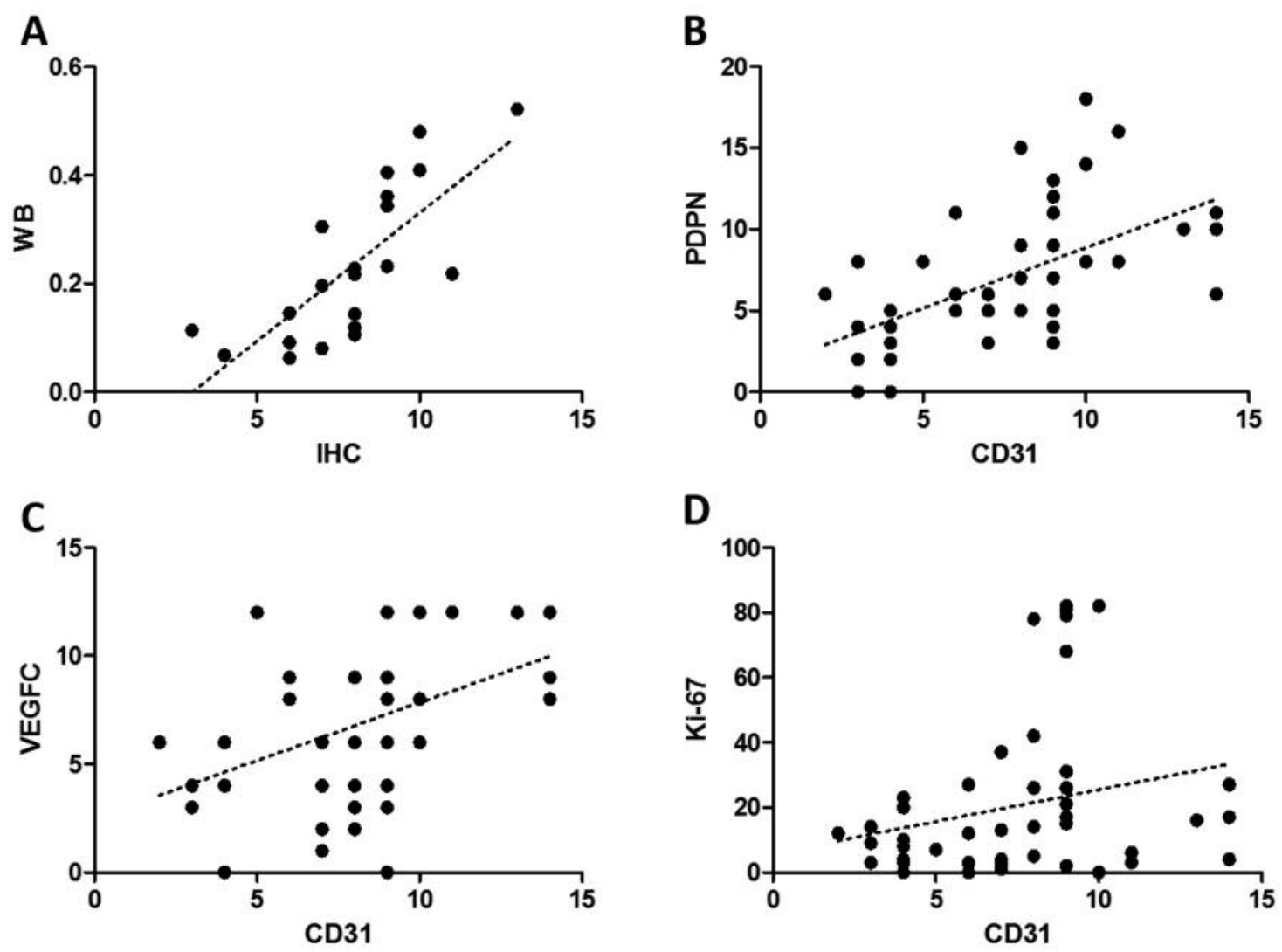

Figure 4. Spearman's correlation test revealed significant positive association between CD31 expression assessed with immunohistochemistry (IHC) and western blot $(W B)(r=0.809, p<0.0001)$ in patients with mycosis fungoides $(A)$. Moreover, strong positive correlations between IHC expression of CD31 vs. (B) podoplanin (PDPN; $r=0.582, p<0.0001)$, (C) vascular endothelial growth factor $C(V E G F C ; r=0.332, p<0.01)$ and $(D) K i-67$ $(r=0.330 ; p<0.01)$ were disclosed.

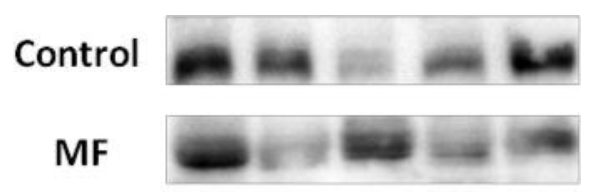

Figure 5. Western blot analysis of expression of CD31 in benign dermatoses (control) and mycosis fungoides $(M F)$ patients.

a VEGF-dependent protein taking part in vascular remodeling, was found increased in MF tissues (22).

In our study, for the first time, we documented a correlation between expression of CD31 and MF advancement. Utilizing IHC and WB, we observed enhanced CD31 expression in advanced compared to early stages (IIB-IVB $v s$. IA-IIA). Similarly, Karpova et al. observed increase in the MVD detected by anti-CD31 antibodies with progression of erythrodermic MF and Sézary syndrome (aggressive variant of CTCL) (23). Concerning skin involvement, an increased CD31 expression in advanced skin lesions comparing to the early ones was revealed. We also noted that infiltration of the skin changes had more important statistical significance than the extent of the lesions in early stages. More abundant expression of CD31 was observed in T2b vs. T2a and T1b vs. T1a. Our results are consistent with recent reports revealing significant differences in terms of prognosis between the disease stages $\mathrm{T} 1 \mathrm{~b} v s$. T1 a and T2b vs. T2a (2). Tumor angiogenesis not only contributes to the growth of tumor mass but also facilitates the spread of neoplastic cells and dissemination of the malignancy. A number of studies have provided data showing associations between increased angiogenesis and dissemination of cancer (24-26). In our study, we observed that the increased CD31 expression positively correlated with nodal involvement. In a variety of solid tumors, as well as hematological malignancies, increased angiogenesis is associated with poor prognosis (27, 28). Surprisingly, in our study, despite the significant correlation between CD31 expression and MF progression, we did not observe any impact on survival. In our opinion, this phenomenon could be explained by the relatively small patient cohort, thus requiring further studies on larger groups of cases. 

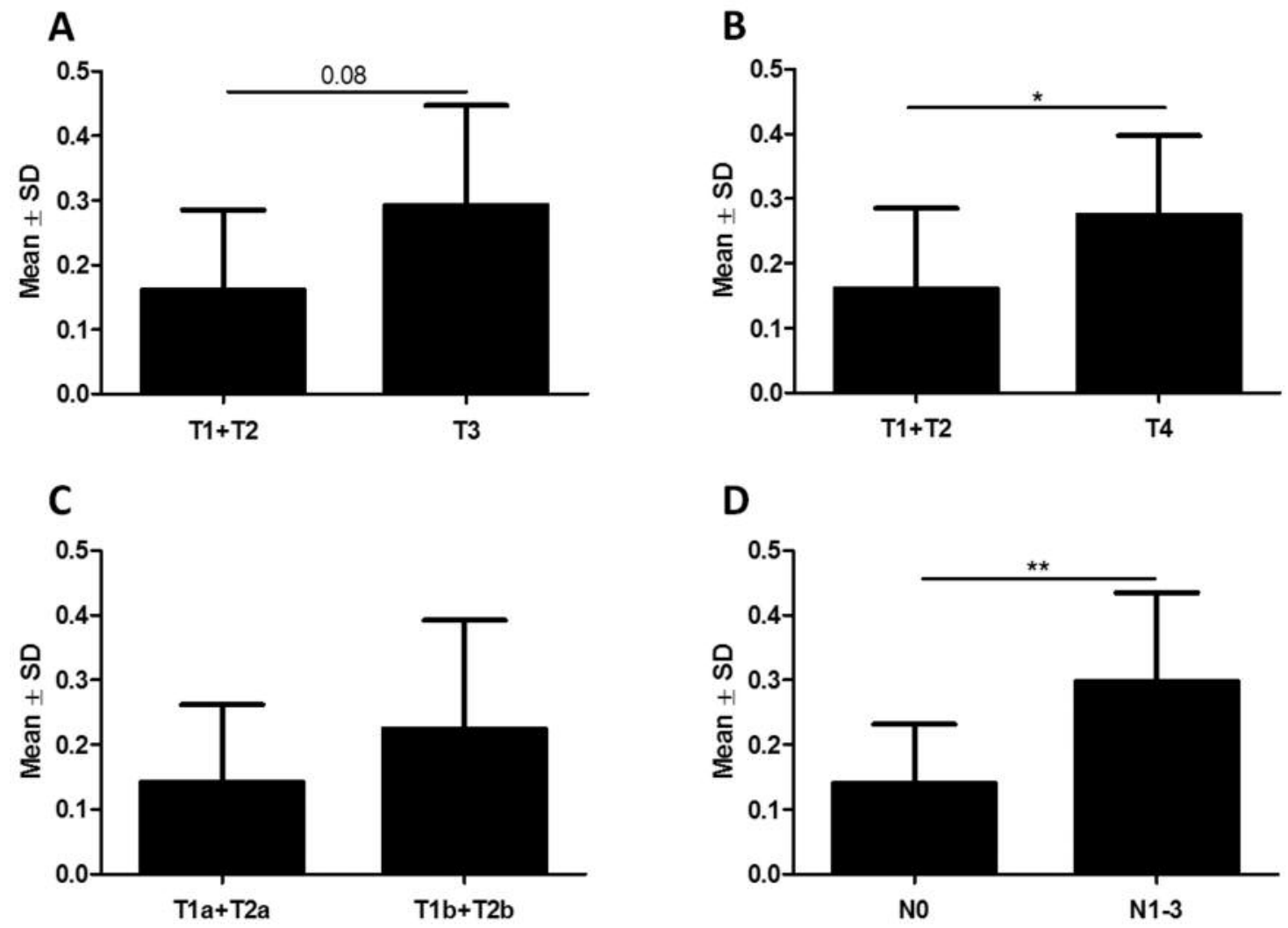

Figure 6. Comparison of CD31 expression assessed with western blot in regard to different stages of skin advancement (A-C) and nodal involvement (D) in mycosis fungoides. SD, Standard deviation. ${ }^{*} p<0.01, * * p<0.0001$, Mann-Whitney test.

Table II. Correlations of immunohistochemical (IHC) and western blot expression of CD31 and podoplanin (PDPN), vascular endothelial growth factor $C(V E G F C)$ and Ki-67 protein in mycosis fungoides patients.

\begin{tabular}{|c|c|}
\hline Correlated markers & $\mathrm{r} ; p$-Value \\
\hline \multicolumn{2}{|l|}{ IHC: } \\
\hline CD31 vs. VEGFC & $\mathrm{r}=0.332 ; p<0.01$ \\
\hline CD31 vs. PDPN & $\mathrm{r}=0.582 ; p<0.0001$ \\
\hline CD31 vs. Ki-67 & $\mathrm{r}=0.330 ; p<0.01$ \\
\hline \multicolumn{2}{|l|}{ WB: } \\
\hline CD31 vs. VEGFC & $\mathrm{r}=0.076 ; p=0.74$ \\
\hline CD31 vs. PDPN & $\mathrm{r}=-0.20 ; p<0.92$ \\
\hline \multicolumn{2}{|l|}{ IHC vs. WB: } \\
\hline CD31 IHC vs. CD31 WB & $\mathrm{r}=0.809 ; p<0.0001$ \\
\hline Ki-67 IHC vs. CD31 WB & $\mathrm{r}=0.09 ; p=0.673$ \\
\hline
\end{tabular}

Significant results are given in bold.

In conclusion, this report demonstrated the expression of CD31 in MF skin biopsies providing new evidence for the role of angiogenesis in the progression, cutaneous and nodal
Table III. Mantel-Cox analysis of survival.

\begin{tabular}{lccc}
\hline & \multicolumn{3}{c}{ Overall survival } \\
\cline { 2 - 4 } & $\mathrm{HR}$ & $95 \%$ CI & $p$-Value \\
\hline IHC: CD31 $(\leq 7 v s .>7)$ & 0.774 & $0.305-1.959$ & 0.588 \\
WB: CD31 $(\leq 0.217 v s .>0.217)$ & 0.955 & $0.191-4.773$ & 0.95 \\
\hline
\end{tabular}

CI, Confidence interval; HR, hazard ratio; IHC, immunohistochemistry; WB, western blot.

involvement in MF. Additionally, our study provided new data for further research on potential future use of CD31 as a new marker of the disease advancement, as well as the target of new therapeutic strategies.

\section{Acknowledgements}

This work was financially supported by the National Science Center (decision no. DEC-2011/01/B/NZ4/01052). 


\section{References}

1 Rakfkiaer E, Cerroni L, Sander CA, Smoller BR and Willemze R: Mycosis fungoides. In: Swerdlow SH, Campo E, Harris NL, Jaffe ES, Pileri SA, Stein H, Thiele J, Vardiman JW, editors. In: WHO classification of tumours of hematopoietic and lymphoid tissues. (ed.) International Agency for Research on Cancer (IARC); Lyon, pp. 269-317, 2008.

2 Olsen E, Vonderheid E, Pimpinelli N, Willemze R, Kim Y, Knobler R, Zackheim H, Duvic M, Estrach T, Lamberg S, Wood G, Dummer R, Ranki A, Burg G, Heald P, Pittelkow M, Bernengo MG, Sterry W, Laroche L, Trautinger F, Whittaker S, ISCL/EORTC. Revisions to the staging and classification of mycosis fungoides and Sezary syndrome: a proposal of the International Society for Cutaneous Lymphomas (ISCL) and the cutaneous lymphoma task force of the European Organization of Research and Treatment of Cancer (EORTC). Blood 110: 17131722, 2007.

3 Vacca A, Moretti S, Ribatti D, Pellegrino A, Pimpinelli N, Bianchi B, Bonifazi E, Ria R, Serio G and Dammacco F: Progression of mycosis fungoides is associated with changes in angiogenesis and expression of the matrix metalloproteinases 2 and 9. Eur J Cancer 33: 1685-1692, 1997.

4 Mazur G, Woźniak Z, Wróbel T, Maj J and Kuliczkowski K: Increased angiogenesis in cutaneous T-cell Lymphomas. Pathol Oncol Res 10: 34-36, 2004.

5 Jankowska-Konsur A, Maj J, Woźniak Z and Baran E: Ocena angiogenezy u chorych na ziarniniaka grzybiastego. Post Dermatol Alergol 26: 186-199, 2009.

6 Bosseila M, Sayed Sayed K, El-Din Sayed SS and Abd El Monaem NA: Evaluation of angiogenesis in early mycosis fungoides patients: dermoscopic and immunohistochemical study. Dermatology 231: 82-86, 2015.

7 Pileri A, Agostinelli C, Righi S, Fuligni F, Bacci F, Sabattini E, Patrizi A, Pileri SA and Piccaluga PP: A vascular endothelial growth factor A (VEGF-A) expression in mycosis fungoides. Histopathology 66: 173-181, 2015.

8 Maj J, Jankowska-Konsur AM, Halon A, Wozniak Z, PlomerNiezgoda E and Reich A: Expression of CXCR4 and CXCL12 and their correlations to the cell proliferation and angiogenesis in mycosis fungoides. Postepy Dermatol Alergol 32: 437-442, 2015.

9 Bachegowda LS and Barta SK: Genetic and molecular targets in lymphoma: implications for prognosis and treatment. Future Oncol 10: 2509-2528, 2014.

10 Chand R, Chandra H, Chandra S and Verma SK: Role of microvessel density and vascular endothelial growth factor in angiogenesis of hematological malignancies. Bone Marrow Res 2016: 5043483, 2016.

11 Andrzejewski W, Kassolik K, Kobierzycki C, Grzegrzolka J, Ratajczak-Wielgomas K, Jablonska K, Halski T, Dziegiel P, Gworys B and Podhorska-Okolow M: Increased skeletal muscle expression of VEGF induced by massage and exercise. Folia Histochem Cytobiol 53: 145-151, 2015.

12 Kerbel R and Folkman J: Clinical translation of angiogenesis inhibitors. Nature Reviews. Cancer 2: 27-739, 2002.

13 Flamme I, Frölich T and Risau W: Molecular mechanisms of vasculogenesis and embryonic angiogenesis. J Cell Physiol 173: 206-210, 1997.

14 Newman PJ and Newman DK: Signal transduction pathways mediated by PECAM-1: new roles for an old molecule in platelet and vascular cell biology. Arterioscler Thromb Vasc Biol 23: 953964, 2003.

15 Woodfin A, Voisin MB and Nourshargh S: PECAM-1: A multifunctional molecule in inflammation and vascular biology. Arterioscler Thromb Vasc Biol 27: 2514-2523, 2007.

16 Privratsky JR and Peter J: Newman PECAM-1: regulator of endothelial junctional integrity. Cell Tissue Res 355: 607-619, 2014.

17 Gumina RJ, Kirschbaum NE, Rao PN, vanTuinen P, Newman PJ: The human PECAM1 gene maps to $17 \mathrm{q} 23$. Genomics 34: 229232, 1996.

18 Jankowska-Konsur A, Kobierzycki C, Reich A, Grzegrzolka J, Bieniek A and Dziegiel P: Expression of SATB1, MTI/II and Ki67 in mycosis fungoides. Anticancer Res 36: 189-197, 2016

19 Jankowska-Konsur A, Kobierzycki C, Grzegrzolka J, Piotrowska A, Gomulkiewicz A, Glatzel-Plucinska N, Reich A, PodhorskaOkołów M, Dziegiel P and Szepietowski JC: Podoplanin expression correlates with disease progression in mycosis fungoides. Acta Derm Venereol In Press, 2016.

20 Jankowska-Konsur A, Kobierzycki C, Reich A, Grzegrzolka J, Maj $\mathrm{J}$ and Dziegiel P: Expression of MCM-3 and MCM-7 in primary cutaneous T-cell lymphomas. Anticancer Res 35: 6017-6026, 2015.

21 Vacca A, Ribatti D, Roncali L, Ranieri G, Serio G, Silvestris F and Dammacco F: Bone marrow angiogenesis and progression in multiple myeloma. Br J Haematol 87: 503-508, 1994.

22 Kawaguchi M, Sugaya M, Suga H, Miyagaki T, Ohmatsu H, Fujita H, Asano Y, Tada Y, Kadono T and Sato S: Serum levels of angiopoietin-2, but not angiopoietin-1, are elevated in patients with erythrodermic cutaneous T-cell lymphoma. Acta Derm Venereol 94: 9-13, 2014.

23 Karpova MB, Fujii K, Jenni D, Dummer R and UrosevicMaiwald M: Evaluation of lymphangiogenic markers in Sézary syndrome. Leuk Lymphoma 52: 491-501, 2011.

24 Zheng CL, Qiu C, Shen MX, Qu X, Zhang TH, Zhang JH and Du JJ: Prognostic impact of elevation of vascular endothelial growth factor family expression in patients with non-small cell lung cancer: an updated meta-analysis. Asian Pac J Cancer Prev 16: 1881-1895, 2015.

25 Zhao DQ, Chen J, Wu YF, Tian D and Zhou RX: Correlation between vascular endothelial growth factor and somatostatin receptor with progression and prognosis in gastric cancer. Hepatogastroenterology 61: 1154-1158, 2014.

26 Bolat F, Kayaselcuk F, Nursal TZ, Yagmurdur MC, Bal N and Demirhan B: Microvessel density, VEGF expression, and tumorassociated macrophages in breast tumors: correlations with prognostic parameters. J Exp Clin Cancer Res 25: 365-372, 2006.

27 Kimura Y, Morohashi S, Yoshizawa T, Suzuki T, Morohashi H, Sakamoto Y, Koyama M, Murata A, Kijima H and Hakamada K: Clinicopathological significance of vascular endothelial growth factor, thymidine phosphorylase and microvessel density in colorectal cancer. Mol Med Rep 13: 1551-1557, 2016.

28 Siamakpour-Reihani S, Owzar K, Jiang C, Turner T, Deng Y, Bean SM, Horton JK, Berchuck A, Marks JR, Dewhirst MW and Alvarez Secord A: Prognostic significance of differential expression of angiogenic genes in women with high-grade serous ovarian carcinoma. Gynecol Oncol 139: 23-29, 2015.

Received July 1, 2016

Revised July 27, 2016 Accepted August 2, 2016 